\title{
ÇEŞME - GERMIYAN KÖYÜ HELLENISTIK ERYTHRAI BRONZ SIKKE DEFINESI
}

\author{
MELIH ARSLAN ${ }^{\circ}$
}

Söz konusu bu define, İzmir İli, Çeșme İlçesi, Germiyan Köyünde, 2002 yılı Mayıs ayında bulunmuş ve Çeşme Müze Müdürlüğü'nce satın alınmıştır. Define, köy içerisinde bir ev inşaatı temeli kazılırken, tesadüfen ortaya çıkmıştır. İnşaat sahibi birkaç para bulmuș ve bunu Köy Muhtarına söylemiş ${ }^{1}$, Muhtar da bu durumu Çeşme Müze Müdürlüğü'ne iletmiștir. Çeşme Müze Müdürlüğü sikkeleri gördüğünde bunun bir define buluntusu olduğuna karar vermiș ve derhal burada bir araștırma bașlatmıștır. Sikkelerin çıktığı alan iyice araştırıldıktan sonra, bu alandan çıkan toprakların da bir elek marifetiyle elenmesi sağlanmıștır. Bu ișlemler sonucunda ve definenin ilk ortaya çıktı̆ında köy halkınca paylașılmıș olanlar da jandarma marifetiyle toplanınca sikke sayısı 228 adet olmuștur (Resim 1, tüm definenin genel görünüşü).

2002 yılı Eylül ayında, Çeşme Müze Müdürlüğü'nde ${ }^{2}$ çalıșımıș olduğum bu bronz define, șimdiye kadar toplu bulummuş Erythrai kenti basımlı sikkelerin en büyük grubunu olușturmaktadır. Aynı zamanda definenin çıkmıș olduğu yerin tam olarak bilinmesi ve sikkelerin bir müze kurtarma kazısı ile toplanmış olması da bu definenin önemini bir kat daha artırmaktadır.

Bu defineden önce, Philip P. Betancourt tarafından 1971 yilında yayınlanmıș "Erythrai'den Bir Bronz Sikke Definesi" isimli bir makale"3 sayesinde bașka bir Erythrai Bronz Sikke Definesi'ni tammaktayız. Betancourt'un

\footnotetext{
+ Anadolu Medeniyetleri Müzesi, 06240 Hisar / ANKARA ; e-mail: arslanmelih@tmet. net.tr.

1 Germiyan Köyü Muhtan ile Çeşme Müze Müdürlüğünde yapmış olduğum bir konuşmada, bu definenin tümünün buradaki kadar olamayacağı ve bir kısım sikkenin ilk bulunduğunda dağılmış olabileceği izlenimini edindiğimi burada belirtmek isterim.

${ }^{2}$ Bu definenin yaymlanmasma izin vermiș olan, Ceșme Müze Müdürü Sn. Emrullah Karaturgut'a burada sonsuz teșekkürlerimi sunmak isterim. Ayrıca, çalıșmalarım esnasında ōzverili yardımların görmüş olduğum, Çeşme Müzesi Arkeoloğu Gamze Aliefendioğlu ve Memur Fevziye Aluok'a teșekkürü bir borç bilirim.

${ }^{3}$ Philip P. Betancourt, "A Hoard of Bronze Coins from Erythrae". ANSMN 17. New York 1971: s. 23-39.
} 
söz konusu yayınındaki sikkeler Amerika'da özel bir koleksiyonda bulunmakta olup, yazarın ifadesi ile define, 1950 yllarında bulunmuş ve daha sonra bir eski eser tüccarı tarafından Amerika Birleșik Devletleri'ne sokulmuş denilmektedir ${ }^{-}$. Bu özel koleksiyonda 42 adet Erythrai sikkesi mevcuttur. Yazar bunları büyük, orta ve küçük bronzlar şeklinde 9 tipte incelemiștir. Amerika'daki definede, bizim Heraklesli sikkelerden 9 adet olup, bunlar Tip V olarak gösterilmiş ve beş farklı memur ismini barındırmaktadır ${ }^{5}$. Bizim Dionysoslu tip dediğimiz bronzlardan ise iki farklı memur ismi taşıyan 2 sikke vardır. Yazar bunları Tip VI olarak göstermiștir "i.

\section{Erythrai Darphanesi:}

İonia Bölgesinin 12 önemli kentinden biri olan Erythrai'nin sikke basımlarn Ephesos, Miletos, Phokai ve Klazomenai'den hemen sonra ve hayli erken bir tarihte bașlamıştur. Şehrin sikke basımları dönem ve karakter farklılıkları göstermesi açısından 9 ayrı seri olarak incelenmelidir.

Kentin 1. Seri sikkeleri, İ.Ö. 6. yüzyılın ortalarından itibaren darp edilmeye bașlanmıștur. Şehrin bu en erken örnekleri elektron olup, Miletos ağırlık biriminde (standarunda) basturılmışur ${ }^{7}$.

2. Seri sikkeler, İ.Ö. 480 yıllarında ortaya çıkmıș olan gümüș didrahmilerdir ${ }^{*}$.

3. Seri sikkeler, İ.Ö. 5. yüzyıl içerisinde görülmeye başlanan, Pers standartundaki gümüș obol ve drahmi serileridir".

4. Seri sikkeler, İ.Ö. 400 civarında ortaya çıkan bronz sikkelerdir. Bunlar Rodos standartunda basılmışlardır ${ }^{10}$.

5. Seri sikkeler, İ. Ö. 4. yüzyıl içinde görülen Rodos standartundaki gümüş drahmi ve tetradrahmi sikkeleridir ${ }^{11}$.

\footnotetext{
' a.g.y., s. 23.

s.g.y., s. 26, no. 25-33.

i. a.g.y., s. 27, no. 34-35.

${ }^{7}$ Bliz, SNG von Aulock, no. 194142.

${ }^{8}$ Bkz., SNG von Aulock, no. 1943.

${ }^{9}$ Bkz., SNG von Aulock, no. 1944-45.

${ }^{10} \mathrm{Bkz}$., SNG von Aulock, no. 1946-47.

11 Bkz., SNG von Aulock, no. 1950-53.
} 
6. Seri sikkeler, İ.Ö. 300-200 yılları arasında ortaya çıkmakta olup, bizim burada yaymmlamakta olduğumuz define sikkeleri de bu grup içinde kalmaktadır. Bu seri Rodos standartında basılmıştır.

7. Seri, İ.Ö. 200-133 yılları arasında basılmıș olan Rodos standartındaki bronzlardir ${ }^{12}$.

8. Seri, İ.Ö. 133 ile Roma İmparatorluk dönemi bașlangıcına kadar tarihlenmiş olan küçük bronzlardır' ${ }^{13}$.

9. Seri ise, Roma İmparatorluk Dönemi basımlanım kapsamaktadır. Kentin imparatorluk sikkeleri, Augustus ile başlar, Gallienus dönemi sonlarına kadar devam eder (İ.Ö. 29 - İ.S. 268). Bu demek oluyor ki kent, İ.Ö. 6. yüzyıldan, İ.S. 3. yüzyll ortalarına kadar süren bir sürede ( 800 yll) kesintişz sikke bastırmıștur. Bundan da kentin uzun yıllar önemini koruduğu ve ekonomik hayatının hep canlı olduğu anlaşılmaktadır.

Yukanda Erythrai Kenti'nin çok kısa sikke basım tarihini özetledikten sonra, esas konumuzu oluşturan, Çeșme Müzesindeki Germiyan Köyü Erythrai Definesine dönecek olursak, definenin 227 bronz ve 1 gümüş sikkeden oluştuğu görülecektir. Bronz sikkeler bilinen birçok yayında, İ. Ö. 300-200 arasına tarihlendirilmektedirler. Yeni yapılan epigrafik çalışmalar ve nümizmatik doktora çalışmaları bu sikkelerin, İ.Ö. 240- 230 tarihlerinde basılmıș olabileceklerini göstermektedir.

Biz bronz sikkelerin tarihlendirilmesini Philip Kinns'in yayımlanmamıs doktora tezine ${ }^{14}$ göre tarihlendirmeyi ve tasnif etmeyi uygun bulmaktayız.

Define'nin bulunduğu Germiyan köyü, antik Erythrai kentinin sorumluluk alanında olmalıdır. Çünkü bugünkü Ildırı Köy merkezini kapsayan antik Erythrai şehri, Germiyan Köyü'ne $8 \mathrm{~km}$. bir mesafededir. Sikkelerin basılmıș olduğu darphane bu kadar yakın bir yerde bulunmuş olan bu define, nümizmatlar ve klasik epigrafi çalışanlarca hiç şüphesiz önemli bir buluntu özelliği taşımaktadır.

Define sikkeleri, 1 gümüs hemidrahmi ve 227 adet Trichalkon olarak isimlendirilen küçük bronz sikkeden oluşmaktadır. İlk yayınlarda bu grup, I. Ö. 300-200 tarihlerine verilmektedir ${ }^{15}$. Daha sonra, Philip Kinns'in doktora

${ }^{12}$ Bkz., SNG von Aulock, no. 1955.

${ }^{13}$ Bkz., SNG von Aulock, no. 1966-67.

${ }^{14}$ Philip Kinns, Studies in the Coinage Ionia: Erythrae, Teos, Lebedus, Colophon, c. 40030 B.C. (Unpublished Doctoral Dissertation. University of Cambridge, 1980): s. 144-8 ve 465-7.

${ }^{15}$ Bkz., BMC Ionia, s. 128-131, no. 104-125 ve SNG von Aulock, no. 1954-1962. 
çalıșmasıyla Erythrai sikkelerinin tarihlendirilme sorunu hemen hemen şimdilik çözümlenmiş olarak kabul görmektedir.

Bir adet olan gümüș hemidrahminin ön yüzünde, genç Herakles'in aslan postu başlıklı portresini görmekteyiz ${ }^{16}$. Arka yüzde, meşe çelengi içinde, EPY yazısı ve altunda bir magistrat (sikke basımından sorumlu memur) monogramı bulunmaktadır. Bu arka yüz kalıbı yeni bir kalıp varyasyonu olup, șimdiye kadar bilinmemektedir. Bu nedenle bu hemidrahmi şimdilik ünik görülmektedir.

Definedeki 227 adet bronz sikkenin arka yüzlerinde yer alan magistrat isimlerinin tümünü daha önceki yayınlardan tanımaktayız. Bronz örnekler iki ana tipten oluşmaktadır. Bu iki grubu, önyüz tiplerine göre, Heraklesli ve Dionysoslu tipler diye simıflandırmaktayz.

Bronz sikkelerin Heraklesli tiplerinin ön yüzünde, genç Herakles'in aslan postu bașlıklı bașı sağa ve arka yüzünde ise, şehrin isim kısaltması EPY ve sikke basımından sorumlu memur isimleri yazılmıștir ${ }^{17}$.

Dionysoslu olarak isimlendirdiğimiz ikinci gruptakilerin ön yüzünde, genç Dionysos'un sarmaşıı yapraklanı ile taçlı başı sağa, arka yüzde, EPY yazısı, üzüm salkımı ve değişen magistrat isimleri yer almaktadır ${ }^{18}$.

Birinci tipi oluşturan Heraklesli sikkelerden definede, 200 adet bulunmaktadır. Bunlar, 12 ayrı magistrat ismi taşımaktadır. Bu grubun sadece 214 sıra nolu sikkesinin magistrat belirlenememiștir. Çünkü sikkenin kondisyonu çok bozuktur.

Dionysos tasvirli sikkelerden definede, 27 adet bulunmaktadır. Bunlar içerisinde, 11 adet magistrat ismi tespit edilmiştir. Magistrat isimleri her iki tip için ortak kullanılmıştır. Fakat, Heraklesli tipin 5. grubunda görmüș olduğumuz, BATAKOS PARAMANOU isimli memur ismini, ne bizim burada yayımlamıș olduğumuz Dionysoslu tipler içerisinde, ne de bașka yayınlanmıș Dionysos örnekler içinde göremediğimizi belirtmek isteriz.

\section{Herakles tipli sikkelerin magistrat gruplan :}

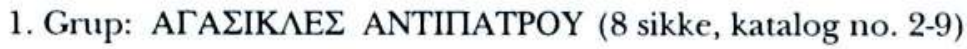

${ }^{16}$ Bkz., BMC Ionia, p. 128, 101.

${ }^{17}$ Bkz., BMC Ionia, p. 129, 104-116 ve SNG von Aulock, 1954-1960.

${ }^{18}$ BMC İonia, 117-125; Weber, 5955; SNG Cop., 658 ve SNG von Aulock, 1961-62. 
2. Grup: АПО $\Lambda \Lambda \Omega N I O \Sigma$ АПО $\Lambda$ ОOTOY (20 sikke, katalog no.10-29)

3. GIтр: АПО $\Lambda \Omega N O \triangle O T O \Sigma$ HPAK $\Lambda$ EITOY (17 sikke, katalog no.3046)

4. Grup: AYTONOMO乏 AYTONOMOY (32 sikke, katalog no.47-78)

5. Grup: BATAKOऽ ПAPAMONOY (9 sikke, katalog no.79-87)

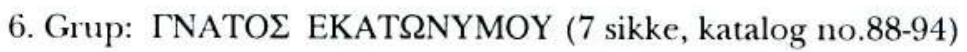

7. Grup: $\triangle \mathrm{AMA} \Lambda \mathrm{H} \Sigma$ APXEANAKTO $\Sigma$ (20 sikke, katalog no.95-114)

8. Grup: $\triangle I O N Y \Sigma I O \Sigma$ IATPOK $\triangle E O Y \Sigma$ (20 sikke, katalog no.115-134)

9. Grup: EPM $\Omega N$ IIOФANTOY (4 sikke, katalog no.135-138)

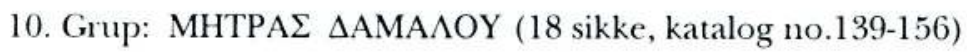

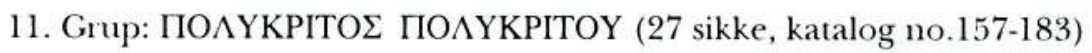

12. Grup: $\Phi \mathrm{I} \Lambda \Omega N$ HPO $\Sigma \Omega N T O \Sigma$ (17 sikke, katalog no.184-200)

Yukarıda, Heraklesli serideki 12 magistrat grubunda görüldüğü üzere, bu gruplar arasında en çok sayıda sikke, 32 adet ile 4 . grupta bulunmaktadır. En az sikke ise, 4 adet sikke ile 9 . grup olmaktadır.

\section{Dionysos tipli sikkelerin magistrat gruplan :}

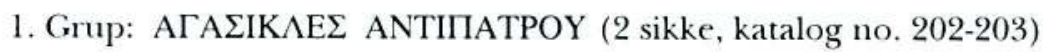

2. Grup: АПO $\Lambda \Omega N I O \Sigma$ АПО $\triangle O \Delta O T O Y$ (3 sikke, kataalog no. 204206)

3. Grup: АПО $\Lambda \Omega N O \triangle O T O \Sigma$ HPAK $\Lambda$ EITOY (2 sikke, katalog no. 207 208)

4. Grup: AYTONOMO乏 AYTONOMOY (1 sikke, katalog no. 209)

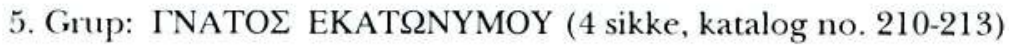

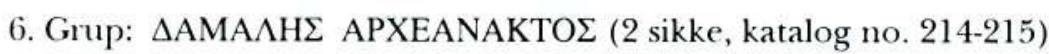

7. Grup: $\triangle I O N Y \Sigma I O \Sigma$ IATPOK AEOY (1 sikke, katalog no. 216)

8. Grup: EPM $\Omega N$ IIOФANTOY (4 sikke, katalog no. 217-220) 


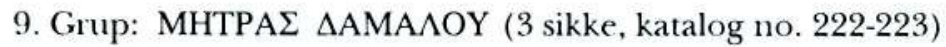

10. Grup: ПОАYКРІTO乏 ПОАYКРITOY (2 sikke, katalog no. 224-225)

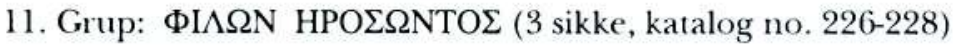

Yukarıda, Dionysoslu serideki 11 magistrat grubunda görüldüğü üzere, bu gruplar arasında en çok sikke sayısı, 4 adet ile, 5. ve 8 . gruplarda bulunmaktadır. En az sikke ise, ladet sikke ile, 4. ve 7. gruplarda bulunmaktadır.

\section{Metroloji :}

Definemizdeki tüm sikkelerin metal ağırlığı, 739.68 gr. gelmektedir. 1 adet gümüs hemidrahmi (2.25 gr.) haricinde 227 adet bronz sikkenin ağırlığı ise, 737.43 gr. çekmektedir. Definedeki bronz sikkeler içinde, en küçük ağırlık, $2.00 \mathrm{gr}$. ile çok aşınmış ve sorumlu memur ismi okunamayan kataloğumuzun 201 nolu sikkesidir. En büyük ağırlık ise, 4.64 gr. ile yine Heraklesli seri içindeki, 88 nolu sikkede bulunmaktadır. Heraklesli seri için ortalama ağırlık, 3.26. gram, Dionysoslu seri için ise, 3.16 gram olarak tespit edilmiştir. En çok rastlanan ağırlık ise, 3.18 gram olanlardır. 12 adet sikkenin bu ağırlıkta olduğu tespit edilmiştir.

Dionysoslu 27 sikke içinde, en düșük ağırlıklı sikke, 2.23 gram, en yüksek ağırlıklısı, 4.11 gramdır. Ortalama ağırlık ise, 3.16 gram gelmektedir. En çok rastlanan ağılık ise, 3.41 gram ile 3 adet sikkede görülmüștür.

\section{Kalıp İlişkileri :}

Definedeki sikkelerin kalıp ilişkilerini de olabildiğince incelemeye çalıştık. Kalıp ilişkilerini, 226 adet bronz sikke üzerinde ve Heraklesli ve Dionysoslu olarak iki bölüme ayırarak yapmak zorundaydık. Fakat, 27 Dionysoslu ve 200 Heraklesli serinin, tüm ön yüzlerinin bir kalıp karşılaștırmasımm sağlıklı yapılamayacağını düşündük. Bu durumda, kalıp karșılaştırmalarını, her bir serinin ortak olan magistrat isimleri arasında uygulanabileceğini gördük. Böylece her iki bronz tipin, 12 ayrı ve 11 ayn grubu olmak üzere, 23 ayrı grubun kendi içerisindeki kalıp ilişkilerini tespit etmeye çalıștık. Her bir grup için kaç ön yüz ve kaç arka yüz kalıbı kullamılmıș olduğu, aynı ve farklı kalıpları ayırt etmek için bir yöntem geliştirdik. Sikkelerin arka yüzünde daima yer alan sorumlu memur isimlerindeki harf karakteri, yani bunlarm 
büyüklükleri, șekilleri, kaligrafileri bize farklı kalıpları seçmemizde çok yardımcı olmuștur. Bunun yanında Dionysoslu serilerde arka yüzde bulunan üzüm salkımmnn yönü, büyüklüğü, sapının sağa veya sola dönük oluşu ve resim stili bu ayrımı yapmamızı daha da kolaylaştırmıştır. Katalog içinde gruplara ayırdığımız sikkelerin, değişen ön yüz ve arka yüz kalıplarını, şu şekildeki kısaltmalarla gösterdik: Ön Yüz Kalıbı ( = ÖYK) ve Arka Yüz Kalıbı (= AYK) kısaltmaları şeklinde ve her değişen kalıbın yanına bir rakam ekledik. Örneğin: ÖYK $2 / A Y K 4$ gibi.

\section{FAYDALI KAYNAKÇA (BIBLIOGRAPHY)}

BMC Ionia

Hunterian

Grose, Fitzwilliam

Kienast 1962

Kinns

Milne 1937

Robert 1933
Barclay V. Head, A Catalogue of The Grek Coins in British Museum.

London, 1892.

Betancourt, Erythrae Philip P. Betancourt, A Hoard of Bronze Coins from Erythrae, ANSMN 17, (New York 1971): 23-39.

G. MacDonald, Catalogue of Greek Coins in the Hunterian Museum, Glasgow. 3 vols. Glasgow, 1901.

S. W. Grose, Catalogue of the McClean Collection of Grek Coins, Fitzwilliam Museum. Cambridge, 1929.

D. Kienast, Literaturüberblicke der griechischen Numismatik, (JNG, 1962): s. 159.

Studies in the Coinage of Ionia: Erythrae, Teos, Lebedus, Colophon, c. 400-30 BC (Unpublished PhD Dissertation, University of Cambridge, 1980), 144-8 and 465-7.

J.G. Milne, Notes on the Oxford Colections, (NC 1937): 153-181.

L. Robert, Inscriptions d'Erythrai, (BCH 57, 1933): 477-481. 
Robert 1936

Robert 1949

SNG Cop.

SNG v. Aulock

Weber
L. Robert, Notes de Numismatique et d'Epigraphie Grecques, (RN, 1936): 271-272.

L. Robert, Monnaies d'Ionie, (Hellenica 7, 1949): 87-88.

Sylloge Nummorum Graecorum, Danish National Museum. Copenhagen, 1946.

Sylloge Nummorum Graecorum Deutschland, Sammlung Hans von Aulock, Ionien. Berlin, 1957.

L. Forrer, The Weber Collection of Greek Coins. 3 vols. London, 1926.

Not: Define sikkelerinin tümü așağıda katalog içerisinde kalıp ilişkileri ile birlikte gösterilmiştir. Resim levhasında ise, memur gruplarından yalnızca birisinin resmi konulmuștur. Resim 1: olarak verdiğimiz resimde definenin toplu olarak genel görünümü sunulmuștur. 


\section{KATALOG}

I.Ö. $300-200$

1. Gümüș Hemidrahmi.

Ö.Y. Genç Herakles'in aslan postu bașlıkı bașı sağa; noktalı bordür.

A.Y. EPY ve sorumlu memur monogramı; meșe çelengi.

Env No.1 ; (2.25 gr. $13 \mathrm{~mm}, 12 \mathrm{~h}$.)

Ref. BMC Ionia, s. 128, no.101 (önyüz benzer).

Herakles Tipli Sikkelerin Kataloğu (No: 2-200)

I. Ö. $240-230$

Ö.Y. Genç Herakles'in aslan postu bașlıklı bașı sağa; noktalı bordür.

A.Y. EPY ve değișen sorumlu memur isimleri.

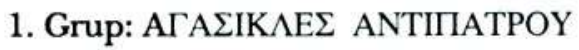

(8 örnek, 5 ÖYK ve 2 AYK farklı, no. 2-9)

2. ÖYK 1 / AYK 1, Env. No. 67; (3.44 gr.; 16 mm.; 12h.)

3. ÖYK 2 / AYK 1, Env. No. 175; (3.13 gr.; 18 mm.; 12h.)

4. ÖYK 3 / AYK 2, Env. No. 48; (2.82 gr.; 17 mm.; 12h.)

5. ÖYK 3 / AYK 2, Env. No. 83; (2.74 gr.; 17 mm.; 12h.)

6. ÖYK 4 / AYK 1, Env. No. 119; (2.76 gr.; 15 mm.; 12h.)

7. ÖYK 4 / AYK 1, Env. No. 174; (2.33 gr.; 16 mm.; 12h.)

8. ÖYK 5 / AYK 1, Env. No. 206; (3.18 gr.; 16 mm.; 12h.)

9. ÖYK 5 / AYK 1, Env. No. 224; (2.69 gr.; 16 mm.; 12h.)

Ref. Kinns, 214. ; BMC Ionia, 103-105. ; SNG von Aulock, 1956.

\section{Grup: АПО $\Lambda \Lambda \Omega N I O \Sigma$ АПO $\Lambda \Lambda O \Delta O T O Y$}

(20 örnek, 6 ÖYK ve 10 AYK farklı, no. 10-29)

10. ÖYK 1 / AYK 1, Env. No. 52; (3.16 gr.; 17 mm.; 5h.)

11. ÖYK 1 / AYK 2, Env. No. 61; (3.12 gr.; 17 mm.; 12h.) 
12. ÖYK 1 / AYK 2, Env. No. 71; (3.26 gr.; 18 mm. 12h.)

13. ÖYK 1 / AYK 3, Env. No. 69; (3.21 gr.; 16 mm.; 12h.)

14. ÖYK 1 / AYK 4, Env. No. 128; (3.26 gr.; 17 mm.; 12h.)

15. ÖYK 2 / AYK 5, Env. No. 60; (3.20 gr.; 17 mm. 12h.)

16. ÖYK 2 / AYK 6, Env. No. 194; (3.01 gr.; 17 mm.; 12h.)

17. ÖYK 2 / AYK 7, Env. No. 202; (3.33 gr.; 17 mm.; 12h.)

18. ÖYK 2 / AYK 7, Env. No. 219; (3.66 gr.; 17 mm.; 4h.)

19. ÖYK 3 / AYK 1, Env. No. 96; (3.36 gr.; 17 mm.; 12h.)

20. ÖYK 3 / AYK 8, Env. No. 183; (3.56 gr.; 17 mm.; 6h.)

21. ÖYK 4 / AYK 8, Env. No. 76; (3.16 gr.; 16 mm.; 5h.)

22. ÖYK 4 / AYK 9, Env. No. 91; (3.37 gr.; 17 mm.; 12h.)

23. ÖYK 4 / AYK 2, Env. No. 95; (3.38 gr.; 17 mm.; 6h.)

24. ÖYK 5 / AYK 10, Env. No. 103; (3.95 gr.; 15 mm.; 5h.)

25. ÖYK 5 / AYK 2, Env. No. 195; (3.24 gr.; 15 mm.; 3h.)

26. ÖYK 6 / AYK 1, Env. No. 108; (3.54 gr.; 17 mm.; 6h.)

27. ÖYK 6 / AYK 9, Env. No. 196; (2.95 gr.; 16 mm.; 6h.)

28. ÖYK 6 / AYK 3, Env. No. 201; (2.55 gr.; 16 mm.; 12h.)

29. ÖYK 6 / AYK 1, Env. No. 228; (3.32 gr.; 17 mm.; 12h.)

Ref. Kinns, 217. ; BMC Ionia, 106.

\section{Grup: АПО $\Lambda \Lambda \Omega N O \triangle O T O \Sigma$ HPAK $\Lambda E I T O \Sigma$} (17 örnek, 9 ÖYK ve 3 AYK farklı, no. 30-46)

30. ÖYK 1 / AYK 1, Env. No. 40; (2.95 gr.; 16 mm.; 12h.)

31. ÖYK 2 / AYK 2, Env. No. 72; (3.77 gr.; 16 mm.; 12h.)

32. ÖYK 3 / AYK 2, Env. No. 121; (4.27 gr.; 17 mm.; 12h.)

33. ÖYK 4 / AYK 3, Env. No. 124; (3.44 gr.; 15 mm.; 12h.)

34. ÖYK 5 / AYK 2, Env. No. 184; (2.78 gr.; 16 mm.; 12h.)

35. ÖYK 6 / AYK 1, Env. No. 38; (3.02 gr.; 16 mm. 12h.)

36. ÖYK 6 / AYK 1, Env. No. 49; (3.27 gr.; 16 mm.; 12h.) 
37. ÖYK 7 / AYK 3, Env. No. 50; (3.14 gr.; 15 mm.; 12h.)

38. ÖYK 7 / AYK 1, Env. No. 63; (3.44 gr.; 17 mm.; 12h.)

39. ÖYK 8 / AYK 1, Env. No. 190; (2.99 gr.; 16 mm. 12h.)

40. ÖYK 8 / AYK 2, Env. No. 205; (3.01 gr.; 16 mm.; 12h.)

41. ÖYK 9 / AYK 2, Env. No. 65; (2.81 gr.; 15 mm.; 12h.)

42. ÖYK 9 / AYK 2, Env. No. 70; (3.40 gr.; 16 mm.; 12h.)

43. ÖYK 9 / AYK 2, Env. No. 74; (3.01 gr.; 16 mm.; 12h.)

44. ÖYK 9 / AYK 3, Env. No. 82; (3.35 gr.; 15 mm; 12h.)

45. ÖYK 9 / AYK 3, Env. No. 168; (2.90 gr. 15 mm.; 12h.)

46. ÖYK 9 / AYK 3, Env. No. 213; (3.35 gr.; 15 mm.; 12h.)

Ref. Kinns, 210. ; BMC Ionia, 107. ; SNG von Aulock, 1958.

\section{Grup: AYTONOMOE AYTONOMOY}

(32 örnek, 2 ÖYK ve 6 AYK farklı, no. 47-78)

47. ÖYK 1 / AYK 1, Env. No. 41; (4.32 gr. 16 mm.; 12h.)

48. ÖYK 1 / AYK 1, Env. No. 106; (3.01 gr. 16 mm.; 6h.)

49. ÖYK 1 / AYK 2, Env. No. 118; (4.02 gr.; 17 mm.; 12h.)

50. ÖYK 1 / AYK 1, Env. No. 140; (3.75 gr.; 16 mm.; 6h.)

51. ÖYK 1 / AYK 3, Env. No. 143; (3.63 gr.; 15 mm.; 6h.)

52. ÖYK 1 / AYK 3, Env. No. 148; (3.77 gr.; 16 mm.; 6h.)

53. ÖYK 1 / AYK 4, Env. No. 155; (4.08 gr.; 16 mm.; 3h.)

54. ÖYK 1 / AYK 2, Env. No. 159; (3.11 gr.; 16 mm.; 6h.)

55. ÖYK 1 / AYK 3, Env. No. 162; (2.48 gr.; 16 mm.; 12h.)

56. ÖYK 1 / AYK 3, Env. No. 164; (3.15 gr.; 15 mm.; 4h.)

57. ÖYK 1 / AYK 4, Env. No. 191; (3.65 gr.; 16 mm.; 3h.)

58. ÖYK 1 / AYK 3, Env. No. 218; (3.60 gr.; 16 mm.; 3h.)

59. ÖYK 2 / AYK 1, Env. No. 31; (3.32 gr.; 15 mm.; 5h.)

60. ÖYK 2 / AYK 3, Env. No. 35; (3.91 gr.; 15 mm.; 8h.)

61. ÖYK 2 / AYK 4, Env. No. 39; (2.76 gr.; 16 mm.; 6h.) 
62. ÖYK 2 / AYK 4, Env. No. 46; (3.21 gr.; 16 mm.; 12h.)

63. ÖYK 2 / AYK 4, Env. No. 88; (3.65 gr.; 16 mm.; 12h.)

64. ÖYK 2 / AYK 2, Env. No. 104; (3.58 gr.; 17 mm.; 6h.)

65. ÖYK 2 / AYK 1, Env. No. 107; (3.08 gr.; 15 mm.; 2h.)

66. ÖYK 2 / AYK 5, Env. No. 115; (2.97 gr.; 15 mm.; 6h.)

67. ÖYK 2 / AYK 1, Env. No. 116; (3.18 gr.; 16 mm.; 12h.)

68. ÖYK 2 / AYK 4, Env. No. 122; (2.73 gr.; 16 mm.; 2h.)

69. ÖYK 2 / AYK 4, Env. No. 123; (2.57 gr.; 16 mm.; 6h.)

70. ÖYK 2 / AYK 3, Env. No. 126; (3.42 gr.; 16 mm.; 12h.)

71. ÖYK 2 / AYK 3, Env. No. 157; (2.95 gr.; 17 mm.; 12h.)

72. ÖYK 2 / AYK 4, Env. No. 170; (2.92 gr.; 17 mm.; 12h.)

73. ÖYK 2 / AYK 2, Env. No. 176; (2.50 gr.; 16 mm.; 6h.)

74. ÖYK 2 / AYK 5, Env. No. 199; (3.16 gr.; 15 mm. 12h.)

75. ÖYK 2 / AYK 5, Env. No. 212; (3.50 gr.; 15 mm.; 2h.)

76. ÖYK 2 / AYK 4, Env. No. 215; (3.99 gr.; 17 mm.; 6h.)

77. ÖYK 2 / AYK 2, Env. No. 222; (2.77 gr.; 16 mm.; 6h.)

78. ÖYK 2 / AYK 5, Env. No. 226; (3.77 gr.; 16 mm.; 5h.)

Ref. Kinns, 218. ; BMC Ionia, 108.

\section{Grup: BATAKOГ ПАPAMONOY}

(9 örnek, 3 ÖYK ve 2 AYK farklı, no. 79-87)

79. ÖYK 1 / AYK 1, Env. No. 44; (3.34 gr.; 16 mm. 12h.)

80. ÖYK 1 / AYK 1, Env. No. 45; (3.08 gr.; 16 mm.; 12h.)

81. ÖYK 2 / AYK 1, Env. No. 62; (3.20 gr.; 17 mm.; 12h.)

82. ÖYK 2 / AYK 1, Env. No. 151; (2.74 gr.; 16 mm.; 12h.)

83. ÖYK 2 / AYK 2, Env. No. 217; (3.49 gr.; 15 mm.; 12h.)

84. ÖYK 3 / AYK 2, Env. No. 84; (3.81 gr.; 15 mm.; 6h.)

85. ÖYK 3 / AYK 2, Env. No. 132; (3.48 gr.; 16 mm.; 6h.)

86. ÖYK 3 / AYK 2, Env. No. 153; (3.18 gr.; 14 mm.; 12h.) 
87. ÖYK 3 / AYK 2, Env. No. 154; (3.84 gr.; 15 mm.; 12h.)

Ref. Kinns, 219 ; BMC Ionia, 109. ; SNG von Aulock, 1957.

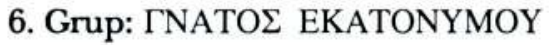

(7 Örnek, 5 ÖYK ve 7 AYK farklı, no. 88-94)

88. ÖYK 1 / AYK 1, Env. No. 32; (4.64 gr.; 16 mm.; 11h.)

89. ÖYK 2 / AYK 4, Env. No. 113; (3.23 gr.; 17 mm.; 12h.)

90. ÖYK 3 / AYK 7, Env. No. 221; (2.64 gr.; 15 mm.; 12h.)

91. ÖYK 4 / AYK 1, Env. No. 59; (4.31 gr.; 16 mm.; 12h.)

92. ÖYK 4 / AYK 3, Env. No. 94; (2.64 gr.; 16 mm.; 12h.)

93. ÖYK 5 / AYK 5, Env. No. 185; (2.71 gr.; 18 mm.; 12h.)

94. ÖYK 5 / AYK 6, Env. No. 216; (3.38 gr.; 17 mm.; 12h.)

Ref. Kinns, 213 ; BMC Ionia, 110. ; SNG von Aulock, 1959.

\section{Grup: $\triangle \mathrm{AMA} \Lambda \mathrm{E} \Sigma$ APXEANAKTO $\Sigma$}

(20 örnek, 10 ÖYK ve 10 AYK farklı, no. 95-114)

95. ÖYK 1 / AYK 1, Env. No. 27; (2.71 gr.; 16 mm.; 12h.)

96. ÖYK 2 / AYK 2, Env. No. 28; (3.18 gr.; 15 mm.; 12h.)

97. ÖYK 3 / AYK 9, Env. No. 178; (3.18 gr.; 16 mm.; 12h.)

98. ÖYK 4 / AYK 6, Env. No. 182; (3.15 gr.; 15 mm.; 12h.)

99. ÖYK 5 / AYK 3, Env. No. 51; (2.77 gr.; 15 mm.; 12h.)

100. ÖYK 5 / AYK 10, Env. No. 189; (2.85 gr.; 16 mm.; 12h.)

101. ÖYK 6 / AYK 10, Env. No. 42; (3.57 gr.; 15 mm.; 12h.)

102. ÖYK 6 / AYK 9, Env. No. 150, (3.73 gr.; 15 mm.; 12h.)

103. ÖYK 7 / AYK 10, Env. No. 109; (2.86 gr.; 16 mm.; 12h.)

104. ÖYK 7 / AYK 5, Env. No. 144; (3.19 gr.; 17 mm.; 6h.)

105. ÖYK 8 / AYK 10, Env. No. 165; (3.18 gr.; 15 mm.; 12h.)

106. ÖYK 8 / AYK 9, Env. No. 220; (2.85 gr.; 15 mm.; 12h.) 
107. ÖYK 9 / AYK 7, Env. No. 78; (3.06 gr.; 15 mm.; 12h.)

108. ÖK 9 / AYK 8, Env. No. 92; (3.58 gr.; 15 mm. 12h.)

109. ÖYK 9 / AYK 10, Env. No. 101; (3.98 gr.; 15 mm.; 12h.)

110. ÖYK 9 / AYK 9, Env. No. 149; (3.60 gr.; 17 mm.; 12h.)

111. ÖYK 10 / AYK 3, Env. No. 114; (3.12 gr.; 16 mm.; 12h.)

112. ÖYK 10 / AYK 8, Env. No. 167; (2.49 gr.; 16 mm.; 12h.)

113. ÖYK 10 / AYK 7, Env. No. 171; (3.06 gr.; 16 mm.; 12h.)

114. ÖYK 10 / AYK 10, Env. No. 179; (3.64 gr.; 15 mm.; 12h.)

Ref. Kinns, 212.; BMC Ionia, 111.; SNG von Aulock, 1960.

\section{Grup: $\triangle \mathrm{IONY}$ IOI IATPOK $\Lambda E O Y \Sigma$}

(20 örnek, 10 ÖYK ve 8 AYK farklı, no. 115-134)

115. ÖYK 1 / AYK 8, Env. No. 47; (3.19 gr.; 17 mm.; 12h.)

116. ÖYK 2 / AYK 6, Env. No. 53; (3.20 gr.; 16 mm.; 12h.)

117. ÖYK 3 / AYK 5, Env. No. 129; (3.54 gr.; 16 mm.; 12h.)

118. ÖYK 4 / AYK 6, Env. No. 137; (2.96 gr.; 15 mm.; 12h.)

119. ÖYK 5 / AYK 4, Env. No. 169; (3.25 gr.; 16 mm.; 12h.)

120. ÖY 6 / AYK 1, Env. No. 58; (3.27 gr.; 16 mm.; 6h.)

121. ÖYK 6 / AYK 8, Env. No. 163; (2.98 gr.; 16 mm.; 12h.)

122. ÖYK 7 / AYK 4, Env. No. 75; (3.39 gr.; 17 mm.; 12h.)

123. ÖYK 7 / AYK 7, Env. No. 135; (3.12 gr.; 17 mm.; 12h.)

124. ÖYK 8 / AYK 6, Env. No. 90; (3.43 gr.; 16 mm.; 12h.)

125. ÖYK 8 / AYK 2, Env. No. 127; (3.54 gr.; 16 mm.; 12h.)

126. ÖYK 9 / AYK 8, Env. No. 43; (3.81 gr.; 17 mm.; 12h.)

127. ÖYK 9 / AYK 6, Env. No. 73; (3.27 gr.; 17 mm.; 12h.)

128. ÖYK 9 / AYK 5, Env. No. 85; (3.88 gr.; 15 mm.; 12h.)

129. ÖYK 10 / AYK 8, Env. No. 33; (3.15 gr.; 17 mm.; 12h.)

130. ÖYK 10 / AYK 7, Env. No. 80; (3.53 gr.; 17 mm.; 6h.)

131. ÖYK 10 / AYK 3, Env. No. 141; (3.81 gr.; 17 mm.; 6 h.) 
132. ÖYK 10 / AYK 7, Env. No. 142; (3.96 gr.; 17 mm.; 6h.)

133. ÖYK 10 / AYK 7, Env. No. 187; (2.58 gr.; 18 mm.; 12h.)

134. ÖYK 10 / AYK 8, Env. No. 188; (4.06 gr.; 18 mm.; 12h.)

Ref. Kinns, 215. ; BMC Ionia, 112.

9. Grup: EPMON $\triangle I O Ф A N T O Y$

(4 örnek, 4 ÖYK ve 4 AYK farklı, no. 135-138)

135. ÖYK 1 / AYK 1, Env. No. 86; (3.32 gr.; 15 mm.; 12h.)

136. ÖYK 2 / AYK 2, Env. No. 105; (4.46 gr.; 16 mm.; 12h.)

137. ÖYK 3 / AYK 3, Env. No. 146; (3.50 gr.; 15 mm.; 12h.)

138. ÖYK 4 / AYK 4, Env. No. 160; (3.47 gr.; 16 mm.; 12h.)

Ref. Kinns, 211. ; BMC Ionia, 113.

\section{Grup: MHTPA $\triangle$ AMA $\Lambda O Y$}

(18 örnek, 4 ÖYK ve 5 AYK farklı, no. 139-156)

139. ÖYK 1 / AYK 1, Env. No. 99; (3.37 gr.; 16 mm.; 11h.)

140. ÖYK 1 / AYK 1, Env. No. 136; (3.43 gr.; 17 mm.; 12h.)

141. ÖYK 1 / AYK 4, Env. No. 147; (3.08 gr.; 15 mm.; 12h.)

142. ÖYK 2 / AYK 1, Env. No. 117; (2.53 gr.; 16 mm.; 12h.)

143. ÖYK 2 / AYK 2, Env. No. 145; (3.00 gr.; 15 mm.; 12h.)

144. ÖYK 2 / AYK 4, Env. No. 173; (2.85 gr.; 16 mm.; 12h.)

145. ÖYK 3 / AYK 2, Env. No. 30; (3.93 gr.; 15 mm.; 12h.)

146. ÖYK 3 / AYK 1, Env. No. 36; (3.64 gr.; 16 mm.; 12h.)

147. ÖYK 3 / AYK 2, Env. No. 131; (2.40 gr.; 16 mm.; 12h.)

148. ÖYK 3 / AYK 2, Env. No. 158; (3.63 gr.; 15 mm.; 12h.)

149. ÖYK 3 / AYK 2, Env. No. 186; (2.89 gr.; 15 mm.; 12h.)

150. ÖYK 3 / AYK 4, Env. No. 197; (4.33 gr.; 16 mm.; 12h.)

151. ÖYK 4 / AYK 3, Env. No. 100; (3.15 gr.; 17 mm.; 12h.) 
152. ÖYK 4 / AYK 3, Env. No. 134; (3.16 gr.; 16 mm.; 11h.) 153. ÖYK 4 / AYK 3, Env. No. 172; (3.23 gr.; 16 mm.; 12h.) 154. ÖYK 4/AYK 3, Env. No. 193; (3.48 gr.; 15 mm.; 11h.) 155. ÖYK 4 / AYK 3, Env. No. 203; (3.30 gr.; 17 mm.; 12h.) 156. ÖYK 4 / AYK 5, Env. No. 223; (3.50 gr.; 17 mm.; 11h.) Ref. Kinns, 207 ; BMC Ionia, 114.

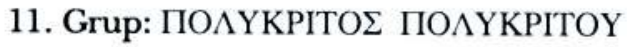

(27 örnek, 6 ÖYK ve 6 AYK farklı, no. 157-183)

157. ÖYK 1 / AYK 1, Env. No. 97; (3.54 gr.; 15 mm.; 12h.) 158. ÖYK 2 / AYK 6, Env. No. 87; (2.53 gr.; 16 mm.; 12h.) 159. ÖYK 2 / AYK 2, Env. No. 208; (3.00 gr.; 17 mm.; 12h.) 160. ÖYK 3 / AYK 6, Env. No. 111; (3.01 gr.; 16 mm.; 12h.) 161. ÖYK 3 / AYK 4, Env. No. 120; (3.64 gr.; 15 mm.; 12h.) 162. ÖYK 3 / AYK 4, Env. No. 200; (3.52 gr.; 16 mm.; 12h.) 163. ÖYK 4 / AYK 4, Env. No. 56; (3.55 gr.; 16 mm.; 12h.) 164. ÖYK 4 / AYK 1, Env. No. 68; (3.09 gr.; 14 mm.; 12h.) 165. ÖYK 4 / AYK 2, Env. No. 156; (3.32 gr.; 17 mm.; 12h.) 166. ÖYK 4 / AYK 6, Env. No. 177; (3.28 gr.; 15 mm.; 12h.) 167. ÖYK 4 / AYK 4, Env. No. 181; (3.50 gr.; 16 mm.; 12h.) 168. ÖYK 4/ AYK 6, Env. No. 198; (3.58 gr.; 15 mm.; 12h.) 169. ÖYK 5 / AYK 1, Env. No. 57; (3.41 gr.; 15 mm.; 12h.) 170. ÖYK 5 / AYK 4, Env. No. 66; (2.68 gr.; 15 mm.; 12h.) 171. ÖYK 5 / AYK 1, Env. No. 89; (2.96 gr.; 16 mm.; 12h.) 172. ÖYK 5 / AYK 6, Env. No. 112; (3.33 gr.; 17 mm.; 12h.) 173. ÖYK 5 / AYK 3, Env. No. 133; (4.45 gr.; 17 mm.; 11h.) 174. ÖYK 5 / AYK 5, Env. No. 204; (3.05 gr.; 15 mm.; 12h.) 175. ÖYK 5 / AYK 3, Env. No. 207; (3.05 gr.; 16 mm.; 12h.) 176. ÖYK 6 / AYK 6, Env. No. 37; (2.65 gr.; 15 mm.; 12h.) 
177. ÖYK 6 / AYK 1, Env. No. 55; (3.33 gr.; 16 mm.; 12h.)

178. ÖYK 6 / AYK 6, Env. No. 130; (2.87 gr.; 17 mm.; 12h.)

179. ÖYK 6 / AYK 3, Env. No. 161; (3.37 gr. 16 mm.; 6h.)

180. ÖYK 6 / AYK 5, Env. No. 180; (3.27 gr.; 15 mm.; 12h.)

181. ÖYK 6 / AYK 5, Env. No. 209; (2.67 gr.; 16 mm. 12h.)

182. ÖYK 6 / AYK 5, Env. No. 210; (3.74 gr. 16 mm.; 12h.)

183. ÖYK 6 / AYK 5, Env. No. 225; (2.86 gr.; 16 mm.; 12h.)

Ref. Kinns, 208; BMC Ionia, 115.

\section{Grup: $\Phi I \Lambda \Omega N$ HPO $\Omega N T O \Sigma$}

(17 örnek, 12 ÖYK ve 5 AYK farklı, no. 184-200)

184. ÖYK 1 / AYK 1, Env. No. 64; (2.89 gr.; 17 mm.; 12h.)

185. ÖYK 2 / AYK 3, Env. No. 77; (2.74 gr.; 17 mm.; 12h.)

186. ÖYK 3 / AYK 5, Env. No. 79; (2.79 gr.; 14 mm.; 12h.)

187. ÖYK 4 / AYK 3, Env. No. 93; (3.09 gr.; 16 mm.; 12h.)

188. ÖYK 5 / AYK 4, Env. No. 98; (3.38 gr.; 15 mm.; 12h.)

189. ÖYK 6 / AYK 5, Env. No. 110; (3.66 gr.; 16 mm., 12h.)

190. ÖYK 7 / AYK 1, Env. No. 227; (3.05 gr.; 16 mm.; 12h.)

191. ÖYK 8 / AYK 4, Env. No. 34; (3.57 gr.; 18 mm., 12h.)

192. ÖYK 8 / AYK 2, Env. No. 166; (2.91 gr.; 15 mm.; 12h.)

193. ÖYK 9 / AYK 5, Env. No. 54; (3.41 gr.; 16 mm., 12h.)

194. ÖYK 9 / AYK 1, Env. No. 125; (3.24 gr.; 15 mm.; 12h.)

195. ÖYK 10 / AYK 2, Env. No. 29; (3.80 gr.; 15 mm., 12h.)

196. ÖYK 10 / AYK 5, Env. No. 211; (3.25 gr.; 16 mm.; 12h.)

197. ÖYK 11 / AYK 4, Env. No. 102; (3.07 gr.; 16 mm.; 12h.)

198. ÖYK 11 / AYK 1, Env. No. 139; (2.20 gr.; 17 mm.; 12h.)

199. ÖYK 12 / AYK 1, Env. No. 138; (3.62 gr. 16 mm.; 12h.)

200. ÖYK 12 / AYK 1, Env. No. 152; (3.22 gr.; 17 mm.; 12h.)

Ref. Kinns, 216. ; BMC Ionia, 116. 
Grubu Bilinmeyen Sikke (No. 201)

201. ÖYK / AYK , Env. No. 214; (2.00 gr.; 16 mm.; 9h.)

Not. Bu sikke çok yıpranmıș ve arka yüz yazılan hiç okunmuyor.

Dionysos Tipli Sikkelerin Kataloğu (No: 202-228)

Ö.Y. Genç Dionysos'un sarmașık yapraklarmulan taç giymiș bașı sağa. A.Y. Sorumlu memur ismi iki veya dört satur olarak, șehir ismi EPY ve sembol olarak üzüm salkımı bazen üstte veya aşağıda yer alır.

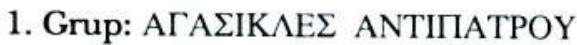

(2 örnek, 1 ÖYK ve 2 AYK farkl, no. 202-203)

202. ÖYK 1 / AYK 1, Env. No. 2; (3.36 gr.; 16 mm.; 12h.)

203. ÖYK 1 / AYK 2, Env. No. 19; (3.12 gr.; 16 mm.; 12h.)

Ref. Kinns, 226. ; BMC Ionia, 117.

\section{Grup: $\mathrm{A \Pi O} \Lambda \Lambda \Omega N I O \Sigma$ АПO $\Lambda$ $\triangle O T O Y$}

(3 örnek, 1 ÖYK ve 2 AYK farklı, no. 204-206)

204. ÖYK 1 / AYK 1, Env. No. 5; (3.41 gr.; 16 mm.; 11h.)

205. ÖYK 1 / AYK 1, Env. No. 81; (3.41 gr. 15 mm. 11h.)

206. ÖYK 1 / AYK 2, Env. No. 11; (3.41 gr. 15 mm.; 11h.)

Ref. Kinns, 229. ; BMC Ionia, 118.; SNG von Aulock, 1961.

\section{Grup: АПО $\Lambda \Lambda \Omega N O \triangle O T O \Sigma$ HPAK $\Lambda$ EITOY}

(2 örnek, 1 ÖYK ve 1 AYK farklı, no. 207-208)

207. ÖYK 1 / AYK 1, Env. No. 12; (3.05 gr.; 16 mm.; 12h.)

208. ÖYK 1 / AYK 1, Env. No. 24; (3.34 gr. 16 mm. 12h.)

Ref. Kinns, 222. ; BMC Ionia, 119. 


\section{Grup: AYTONOMOE AYTONOMOY}

(1 örnek, 1 ÖYK ve 1 AYK farklı, no. 209)

209. ÖYK 1 / AYK 1, Env. No. 6; (4.11 gr.; 16 mm.; 10h.)

Ref. Kinns, 230. ; BMC Ionia, 120.

\section{Grup: $\Gamma N \Omega T O \Sigma$ EKAT $\Omega N Y M O Y$}

(4 örnek, 4 ÖYK ve 4 AYK farklı, no. 210-213)

210. ÖYK 1 / AYK 1, Env. No. 8; (3.66 gr.; 16 mm.; 12h.)

211. ÖYK 2 / AYK 2, Env. No. 17; (2.88 gr.; 17 mm.; 12h.)

212. ÖYK 3 / AYK 3, Env. No. 20; (2.91 gr.; 16 mm.; 12h.)

213. ÖYK 4 / AYK 4, Env. No. 21; (3.54 gr.; 17 mm.; 12h.)

Ref. Kinns, 225. ; BMC Ionia, 122.

6. Grup: $\triangle$ AMA $\triangle E H \Sigma$ APXEANAKTO

(2 örnek, 2 ÖYK ve 2 AYK farklı, no. 214-215)

214. ÖYK 1 / AYK 1, Env. No. 13; (3.12 gr.; 14 mm.; 5h.)

215. ÖYK 2 / AYK 2, Env. No. 23; (2.55 gr.; 15 mm.; 6h.)

Ref. Kinns, 224. ; Weber, 5955.

\section{Grup: $\triangle \mathrm{IONY} \Sigma \mathrm{IO} \Sigma$ IATPOK $\Lambda$ EOY $\Sigma$}

(1 örnek, 1 ÖYK ve 1 AYK farklı, no. 216)

216. ÖYK 1 / AYK 1, Env. No. 22; (3.14 gr.; 16 mm.; 12h.)

Ref. Kinns, 227. ; BMC Ionia, 123.

\section{Grup: EPMON $\triangle I O \Phi A N T O Y$}

(4 örnek, 2 ÖYK ve 4 AYK farkh, no. 217-220) 
217. ÖYK 1 / AYK 1, Env. No. 14; (2.79 gr.; 14 mm.; 12h.) 218. ÖYK 1 / AYK 2, Env. No. 18; (2.61 gr.; 15 mm.; 12h.) 219. ÖYK 2 / AYK 3, Env. No. 26; (2.42 gr.; 14 mm.; 12h.) 220. ÖYK 2 / AYK 4, Env. No. 192; (4.10 gr.; 15 mm.; 12h.) Ref. Kinns, 223, ; BMC Ionia, 124.

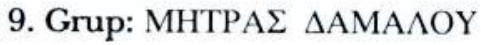

(3 örnek, 1 ÖYK ve 2 AYK farklı, no. 221-223)

221. ÖYK 1 / AYK 1, Env. No. 3; (3.64 gr.; 17 mm.; 12h.) 222. ÖYK 1 / AYK 2, Env. No. 4; (2.94 gr.; 15 mm.; 11h.) 223. ÖYK 1 / AYK 2, Env. No. 10; (2.97 gr.; 16 mm.; 12h.) Ref. Kinns, 219. ; Weber 5956.

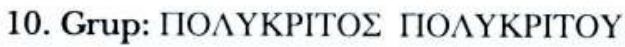

(2 örnek, 2 ÖYK ve 2 AYK farkl, no. 224-225)

224. ÖYK 1 / AYK 1, Env. No. 9; (2.23 gr.; 15 mm.; 12h.) 225. ÖYK 2 / AYK 2, Env. No. 25; (2.45 gr.; 14 mm.; 12h.) Ref. Kinns, 220. ; BMC Ionia, 125.

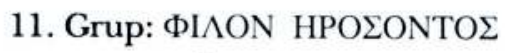
(3 örnek, 1 ÖYK ve 2 AYK farkl, no. 226-228)

226. ÖYK 1 / AYK 1, Env. No. 7; (3.33 gr.; 15 mm.; 12h.) 227. ÖYK 1 / AYK 1, Env. No. 16; (2.77 gr.; 15 mm.; 12h.) 228. ÖYK 1 / AYK 2, Env. No. 15; (3.89 gr.; 14 mm.; 12h.) Ref. Kinns, 228. ; SNG Cop., 658. 